\title{
МОДЕЛИРОВАНИЕ ЭТАПА РАЗВИТИЯ ТЕЧЕНИЯ ВЯЗКОПЛАСТИЧНОЙ СРЕДЫ ПО НАКЛОННОЙ ПЛОСКОСТИ 1
}

\section{MODELING OF THE STAGE OF DEVELOPMENT OF THE FLOW OF A VISCOPLASTIC MEDIUM ALONG AN INCLINED PLANE}

\section{Andreichenko M. Safronchik}

Summary. The development of the flow of a viscoplastic medium along an inclined plane under the influence of gravity from a state of rest is considered. The flow zone grows gradually, its boundary is unknown in advance and is determined in the process of solving the problem. The angle of inclination is chosen in such a way that the so-called "slipping" effect along the solid wall appears over time. The problem is posed within the framework of the five - parameter Slibar-Paslay model, which allows taking into account the difference in the behavior of the material during loading and unloading, and the possible "slipping" of the material along a solid wall is taken into account using a model of a smooth transition from sticking to slipping similar to the hypothesis of prof. N.P. Petrov for a viscous liquid. The solution of the problem is constructed using the Galerkin projection method using orthogonal Chebyshev polynomials of the first kind as basis functions.

Keywords: unsteady flow; mathematical and computer modeling; nonNewtonian fluids; viscoplastic materials; multiphase flows.
Андрейченко Дмитрий Константинович

Д.ф.-м.н., профессор, Саратовский национальный исследовательский государственный университет имени Н.Г. Чернышевского, г. Саратов

Сафрончик Мария Ильинична

Аспирант, Саратовский национальный исследовательский государственный университет имени Н.Г. Чернышевского, г. Саратов safronchikmi@yandex.ru

Аннотация. Рассматривается развитие течения вязкопластичной среды по наклонной плоскости под действием силы тяжести из состояния покоя. Зона течения растет постепенно, её граница заранее неизвестна и определяется в процессе решения задачи. Угол наклона выбирается таким образом, чтобы с течением времени проявился так называемый эффект «проскальзывания» вдоль твердой стенки. Задача ставится в рамках пятипараметрической модели Слибара — Паслая, позволяющей учесть различие в поведении материала при нагружении и разгрузке, а возможное «проскальзывание» материала вдоль твердой стенки учитывается с помощью модели плавного перехода от прилипания к проскальзыванию аналогичной гипотезе проф. Н.П. Петрова для вязкой жидкости. Решение задачи строится с помощью проекционного метода Галеркина с использованием в качестве базисных функций ортогональных полиномов Чебышёва первого рода.

Ключевые слова: нестационарное течение; математическое и компьютерное моделирование; неньютоновские жидкости; вязкопластические материалы; многофазные течения.

Анализ нестационарных режимов течения представляет определенные математические трудности, так как границы раздела зон течения изменяются во времени и в определяющих соотношениях среды отсутствует информация о распределении напряжений в твердой зоне. Таким образом при исследовании нестационарных течений приходится решать начально-краевые задачи с вырождением области начального существования фазы в особую точку так как область течения отсутствует в начальный момент.

Так же экспериментальные исследования выявили еще ряд особенностей в реодинамике вязкопластич- 


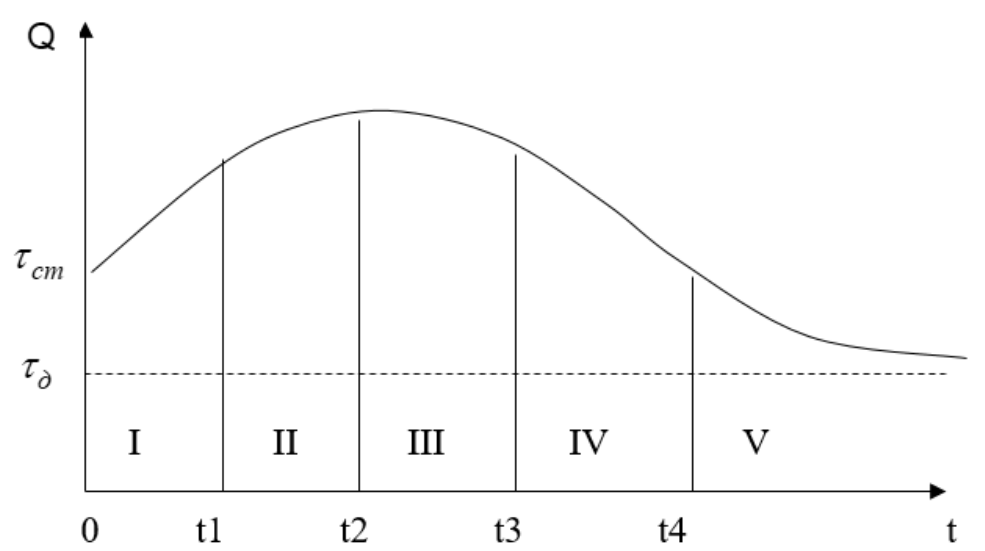

Рис. 1. Характерные временные этапы развития и затухания течения I - разрушение структуры без "пристенного скольжения"

II - разрушение структуры со скольжением

III - переходный этап со скольжением

IV - переходный этап без скольжения

V - восстановление структуры

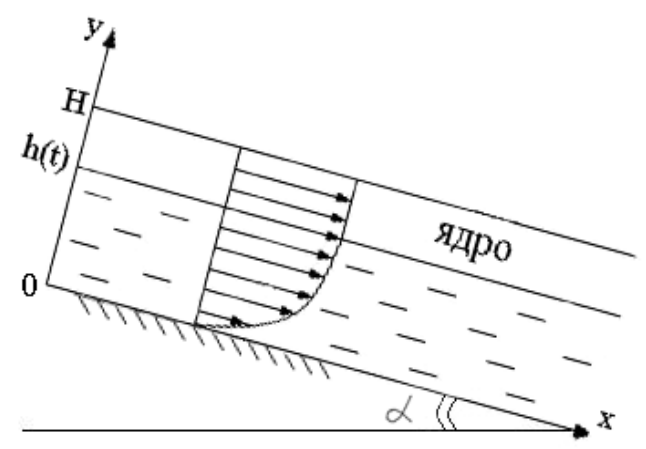

Рис. 2. Схема течения по наклонной плоскости

ных сред таких как различие в поведении материала при нагружении и разгрузке: разрушение материала происходит при одном пределе текучести, а восстановление структуры при другом, значительно меньшем, а также аномальное поведение вязкопластической жидкости у твердых стенок, заключающимся в резком изменении сопротивления материала при определенных скоростях движения. Это явление получило название «эффекта пристенного скольжения».

При воздействии на вязкопластичную среду немонотонной нагрузкой, можно выделить несколько характерных временных этапов её поведения [1, с. 176]. Примерная схема развития и затухания течения изображена на рисунке 1. По оси X время, по оси Y нагрузка.

\section{Постановка заАачи}

Пусть слой вязкопластичной среды толщины Н находится на горизонтальной плоскости. В момент $\mathrm{t}=0$ плоскость была наклонена на угол а к горизонту. Область течения развивается постепенно при достижении касательным напряжением статического предела текучести и граница этой области заранее неизвестна. Течение считается плоскопараллельным, если размеры плоскости достаточно велики. Направим ось ОХ вдоль плоскости, а ось OY перпендикулярно потоку, как показано на рисунке 2 [2, с. 137].

Постановка задачи дается в рамках пятипараметрической модели Слибара - Паслая, которая для случая чистого сдвига имеет вид [3].

$$
\begin{array}{rlrl}
\text { Нагружение } & \text { Разгрузка } \\
\tau-\tau_{d}=\eta\left|\frac{\partial V}{\partial n}\right| \operatorname{sign} \frac{\partial V}{\partial n} \quad \text { nри } \tau>\tau_{s} & \tau-\tau_{d}=\eta\left|\frac{\partial V}{\partial n}\right| \operatorname{sign} \frac{\partial V}{\partial n} \quad \text { nри } \tau>\tau_{d} \\
0 & =\frac{\partial V}{\partial n} \text { nрu } \tau \leq \tau_{s} & 0 & =\frac{\partial V}{\partial n} \quad \text { npu } \tau \leq \tau_{d}
\end{array}
$$

где $\tau_{s}$ - статический предел текучести, $\tau_{d}$ - динамический предел текучести, $\eta$ - структурная вязкость, $V$ скорость, $n-$ нормаль к направлению скорости. 


\section{Изменение подвижной границы течения при его развитии}

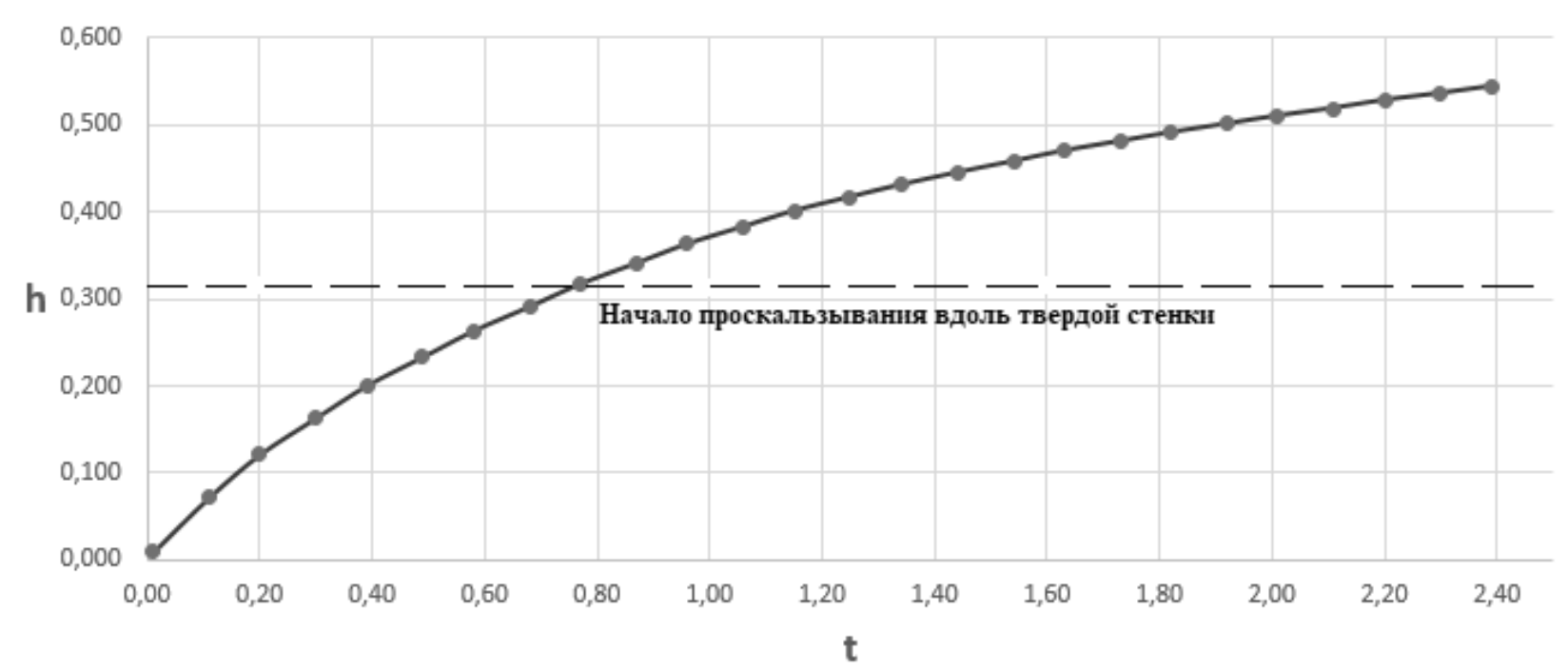

Рис. 3. Изменение границы зоны течения

Величина угла наклона плоскости выбирается такой, чтобы с течением времени появился эффект «проскальзывания», для учета которого используется гипотеза, аналогичная гипотезе проф. Н.П. Петрова для вязкой жидкости [4]. Согласно этой гипотезе, сопротивление на твердой стенке падает и материал начинает проскальзывать вдоль неё при достижении касательным напряжением некоторого критического значения $\tau^{*}$. Скорость скольжения предлагается считать пропорциональной разнице между фактическим и критическим напряжениями

$$
\begin{aligned}
& \tau-\tau^{*}=\lambda\left|V_{\text {fluid }}-V_{\text {wall }}\right|, \quad \tau>\tau^{*} \\
& V_{\text {fluid }}=V_{\text {wall }}, \quad \tau \leq \tau^{*}
\end{aligned}
$$

при этом коэффициент $\lambda$ можно условно назвать коэффициентом «внешнего» трения вязкопластичной среды о твердую стенку. Для данной задачи соотношение между параметрами в рамках пятипараметрической модели будет следующим: $g \rho H \sin (\alpha)>\tau^{*}>\tau_{s}$.

Для единственной компоненты скорости краевая задача имеет вид:

$$
\begin{aligned}
& \frac{\partial V_{x}}{\partial t}=v \frac{\partial^{2} V_{x}}{\partial y^{2}}+g \sin \alpha, \quad 0<y<h(t), \quad 0<t \leq T_{1} \\
& V_{x}(y, 0)=0, \quad h(0)=0 \\
& V_{x}(0, t)= \begin{cases}0, & 0<t \leq t \\
\left(\tau(0, t)-\tau^{*}\right) / \lambda, & t^{*}<t \leq T_{1}\end{cases} \\
& \left(\frac{\partial V_{x}}{\partial y}\right)_{y \rightarrow h(t)-0}=\frac{\tau_{s}-\tau_{d}}{\eta}
\end{aligned}
$$

$$
\begin{aligned}
& \left.V_{x}\right|_{y \rightarrow h(t)-0}=U(t), \text { где } \\
& U(t)=g t \sin \alpha-\frac{\tau_{s}}{\rho} \int_{0}^{t} \frac{d \xi}{H-h(\xi)}
\end{aligned}
$$

где $v=\eta / \rho-$ аналог кинематической вязкости, $U(t)$ - скорость ядра течения. Вводя новые переменные по формулам:

$$
z=H y / h(t), \quad V_{x}(y, t)=V_{x}(z, t)
$$

получим краевую задачу в области с постоянными границами $0<z<H, t>0$, (аналог уравнений (7) и (8) представлен в дифференциальной форме)

$$
\begin{aligned}
& \frac{\partial^{2} V_{x}}{\partial z^{2}}=\frac{h^{2}(t)}{v H^{2}}\left[\frac{\partial V_{x}}{\partial t}-z \frac{h^{\prime}(t)}{h(t)} \frac{\partial V_{x}}{\partial z}-g \sin \alpha\right] \\
& V_{x}(z, 0)=0, \quad h(0)=0 \\
& V_{x}(0, t)= \begin{cases}0, & 0<t \leq t \\
\left(\tau(0, t)-\tau^{*}\right) / \lambda, & t^{*}<t \leq T_{1}\end{cases} \\
& \left(\frac{\partial V}{\partial z}\right)_{z=H}=\frac{h(t)}{H} \frac{\tau_{s}-\tau_{d}}{\eta} \\
& \frac{v H^{2}}{h(t)} \frac{\partial^{2} V_{x}(H, t)}{\partial z^{2}}+H h^{\prime}(t) \frac{\partial V_{x}(H, t)}{\partial z}=-\frac{\tau_{s} h(t)}{\rho(H-h(t))}
\end{aligned}
$$

\section{Построение решения}

Начально-краевая задача (3) - (8) в области с неизвестной подвижной границей принадлежит к классу 
Профиль скорости при развитии течения

7,00000

6,00000

$\mathrm{V}(\mathrm{t}, \mathrm{z})$

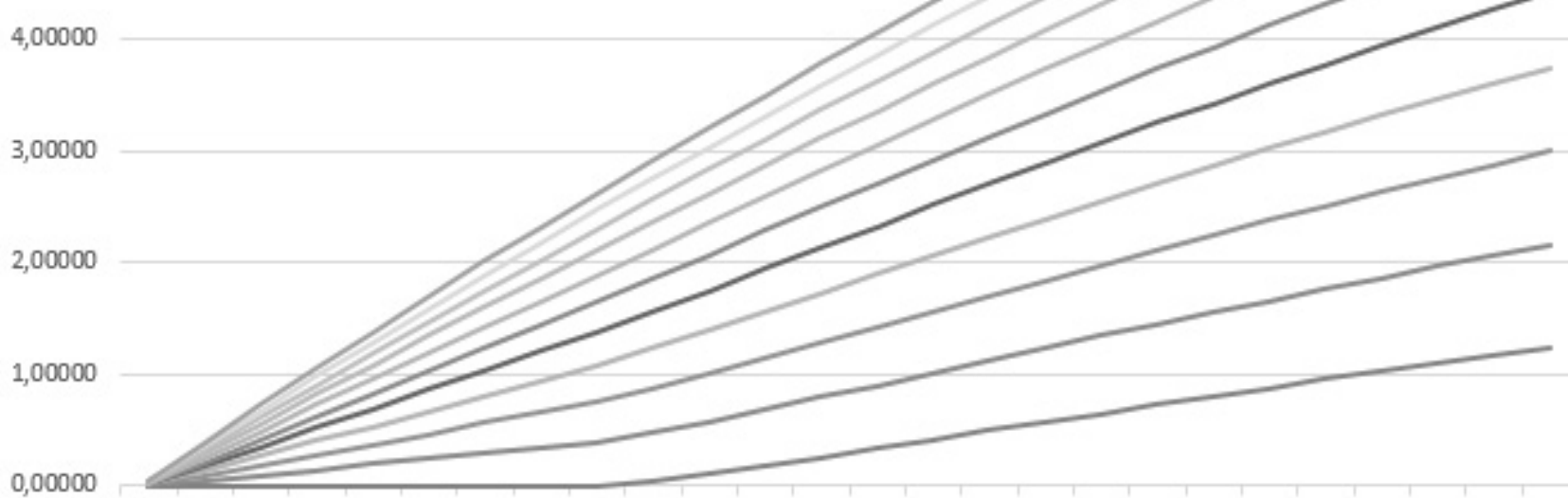

$-1,00000$
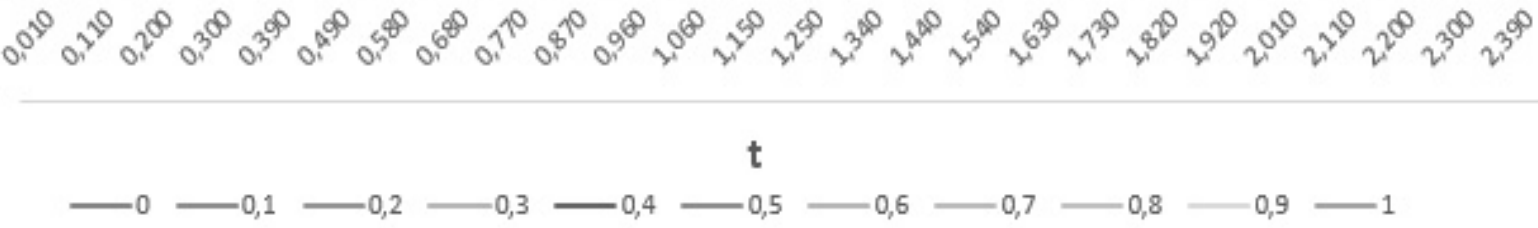

Рис. 4. Профиль скорости при развитии течения

не вполне корректных задач, т.к. в начальный момент область течения отсутствует. Как следствие, требование отсутствия особенностей решения начально-краевой задачи (10) - (14) при $t \rightarrow+0$ приводит к асимптотическому представлению

$$
\begin{aligned}
& h(t)=A t+\overline{\bar{o}}(t), V(z, t)=B z t+B_{1} z t^{2}\left(1-\frac{z}{2 H}\right)+\overline{\bar{o}}(t) \\
& t \rightarrow 0 \\
& A=\frac{\eta}{\tau_{s}-\tau_{d}}\left[g \sin \alpha-\frac{\tau_{s}}{\rho H}\right], B=\frac{1}{H}\left[g \sin \alpha-\frac{\tau_{s}}{\rho H}\right] \\
& B_{1}=\frac{A^{2}}{v H} g \sin \alpha
\end{aligned}
$$

Для решения задачи применяется проекционный метод Бубнова- Галеркина с использованием в качестве базисных функций ортогональных полиномов Чебышёва первого рода. Представим функцию $V_{x}(z, t)$ в виде ряда по ортогональным полиномам Чебышёва:

$$
V(z, t) \approx \sum_{n=0}^{N+2} V_{n}(t) T_{n}\left(2 \frac{z}{H}-1\right),
$$

$$
\mathrm{T}_{n}(z)=\cos (n \arccos z)
$$

Представим (10) в виде:

$\mathbb{F}[V]=-\frac{\partial^{2} V}{\partial z^{2}}+\frac{h^{2}(t)}{v H^{2}}\left[\frac{\partial V}{\partial t}-z \frac{h^{\prime}(t)}{h(t)} \frac{\partial V}{\partial z}-g \sin \alpha\right]=0$

из (12) и (16) следует

$$
\sum_{n=0}^{N+2}(-1)^{n} V_{n}(t)= \begin{cases}0, & 0<t \leq t^{*} \\ \left(\tau(0, t)-\tau^{*}\right) / \lambda, & t^{*}<t\end{cases}
$$

из (13) и (16)

$\sum_{n=1}^{N+2} n^{2} V_{n}(t)=\frac{h(t)}{2} \frac{\tau_{s}-\tau_{d}}{\eta}$

из (14) и (16) 


$$
\frac{4 v}{3 h} \sum_{n=0}^{N+2} n^{2}\left(n^{2}-1\right) V_{n}(t)+2 h^{\prime}(t) \sum_{n=1}^{N+2} n^{2} V_{n}(t)=-\frac{\tau_{s} h(t)}{\rho(H-h(t))}
$$

Для того, чтобы приближенно выполнить уравнение (16), требуем:

$$
\begin{aligned}
& \int_{0}^{H} \mathbb{F}[V] T_{n}\left(2 \frac{z}{H}-1\right) d z=0, \mathrm{n}=\overline{0, N} \\
& A_{n, m}^{(0)}=\int_{-1}^{1} T_{n}(x) T_{m}(x) d x \\
& A_{n, m}^{(k)}=\int_{-1}^{1} T_{n}(x) T_{m}^{(k)}(x) d x \\
& -\left(\frac{2}{H}\right)^{2} \sum_{m=0}^{N+2} A_{n, m}^{(2)} V_{m}(t)+\frac{h^{2}(t)}{v H^{2}} \sum_{m=0}^{N+2} A_{n, m}^{(0)} V_{m}^{\prime}(t)- \\
& -\frac{h(t) h^{\prime}(t)}{2 v H} \sum_{m=0}^{N+2}\left(A_{n, m}^{(1)}+\frac{1}{2}\left(A_{n+1, m}^{(1)}+A_{n-1, m}^{(1)}\right)\right) V_{m}(t)- \\
& -\frac{h^{2}(t)}{v H^{2}} A_{n, 0}^{(0)} g \sin \alpha=0
\end{aligned}
$$

(18), (19), (23) представляет собой записанную в неявной форме систему обыкновенных дифференциальных уравнений первого порядка. Приведение её к нормальной форме Коши

$$
\mathbf{Y}^{\prime}=\mathbf{F}(t, \mathbf{Y}), \quad \mathbf{Y}=\left(V_{0}, V_{1}, \ldots, V_{N}, h\right)^{T}
$$

и нахождение величины $Y^{\prime}$ по известным в текущий момент времени $t$ величинам $Y$ выполняется в следующей последовательности.

Из (18) и (19) находим $V_{N+1}$ и $V_{N+2}$ по $V_{0}, V_{1}, \ldots, V_{N}$ и $h$, из (20) находим $h^{\prime}(t)$ Далее продифференцируем (18) и (19) по t.

$$
\begin{aligned}
& \sum_{n=0}^{N+2}(-1)^{n} V_{n}^{\prime}(t)= \begin{cases}0, & 0<t \leq t^{*} \\
\left(\tau^{\prime}(0, t)-\tau^{*}\right) / \lambda, & t^{*}<t\end{cases} \\
& \sum_{n=0}^{N+2} n^{2} V_{n}^{\prime}(t)=\frac{h^{\prime}(t)}{2} \frac{\tau_{s}-\tau_{d}}{\eta}
\end{aligned}
$$

Уравнения (25), (26) и (23) представляют собой систему линейных алгебраических уравнений относительно $V_{1}, \ldots, V_{N}, V_{N+1}, V_{N+2}$ Начальные условия для системы обыкновенных дифференциальных уравнений (23) следуют из асимптотического представления (15) при $t=t_{0}$, $0<t_{0}<<1$.

\section{Результаты молемирования}

Моделирование развития течения вязкопластичной среды по наклонной плоскости проводится со следующими параметрами $\tau_{d}=0.5 \tau_{s}, \tau_{s}=0.5 \tau$, $\rho=900$, $\eta=128, H=1, \lambda=500$.

На рисунке 3 показано изменение границы зоны течения, в момент $t^{*}=0,77$ (пунктирная линия на графике) начинается проскальзывание вдоль твердой стенки и скорость роста зоны течения замедляется.

На рисунке 4 показан профиль скорости при развитии течения. В момент $\mathrm{t}=0,77$ происходит отрыв от твердой стенки, граничные условия в (25) меняются и скорость В точке $z=0$ начинает расти.

\section{Зак^ючение}

Компьютерное моделирование в задачах развития течения вязкопластических сред эффективно реализуется на основе: 1) предварительного отображения области с подвижной границей на область с неподвижной границей; 2) дискретизации преобразованной начально-краевой задачи по независимой пространственной переменной на основе проекционного метода Бубнова-Галеркина с использованием в качестве базисных функций смещенных ортогональных полиномов Чебышева 1-го рода; 3) численного интегрирования полученной в результате задачи Коши для системы обыкновенных дифференциальных уравнений. При этом в математических моделях развития течений корректная постановка начальных условий для соответствующих начально-краевых задач обеспечивается предварительным применением методов асимптотического интегрирования. Данный метод позволяет моделировать динамику поля скоростей среды и движение границы раздела, а также находить моменты времени, соответствующие началу проскальзывания среды. Однако для осесимметричных задач требуется развитие предложенного метода, связанное с наличием особых точек на оси симметрии.

\section{ЛИТЕРАТУРА}

1. Сафрончик А.И. Неустановившиеся течения вязкопластичной среды с учетом пристенного скольжения и «запаздывания» восстановления структуры // Математика. Механика: сб. научных трудов.— Вып. 2. — Саратов: Изд-во Сарат. ун-та, 2000. — С. 174-177. 
2. Сафрончик М.И. Развитие течения вязкопластичной среды по наклонной плоскости // Математика. Механика: сб. научных трудов. - Вып. 11.—Саратов: Изд-во Сарат. ун-та, 2009.-С. 136-140.

3. Slibar A., Paslay P.R. Retarded Flow of Bingam Materials // J. of Appl.Mech. — 1959 March. — P. 107-112.

4. Петров Н.П. Гидродинамическая теория смазки // Сб. под редакцией проф. Лейбензона Л.С. ГТТИЗ - М., 1934.— С. 245.

(с) Андрейченко Дмитрий Константинович, Сафрончик Мария Ильинична ( safronchikmi@yandex.ru ).

Журнал «Современная наука: актуальные проблемы теории и практики»

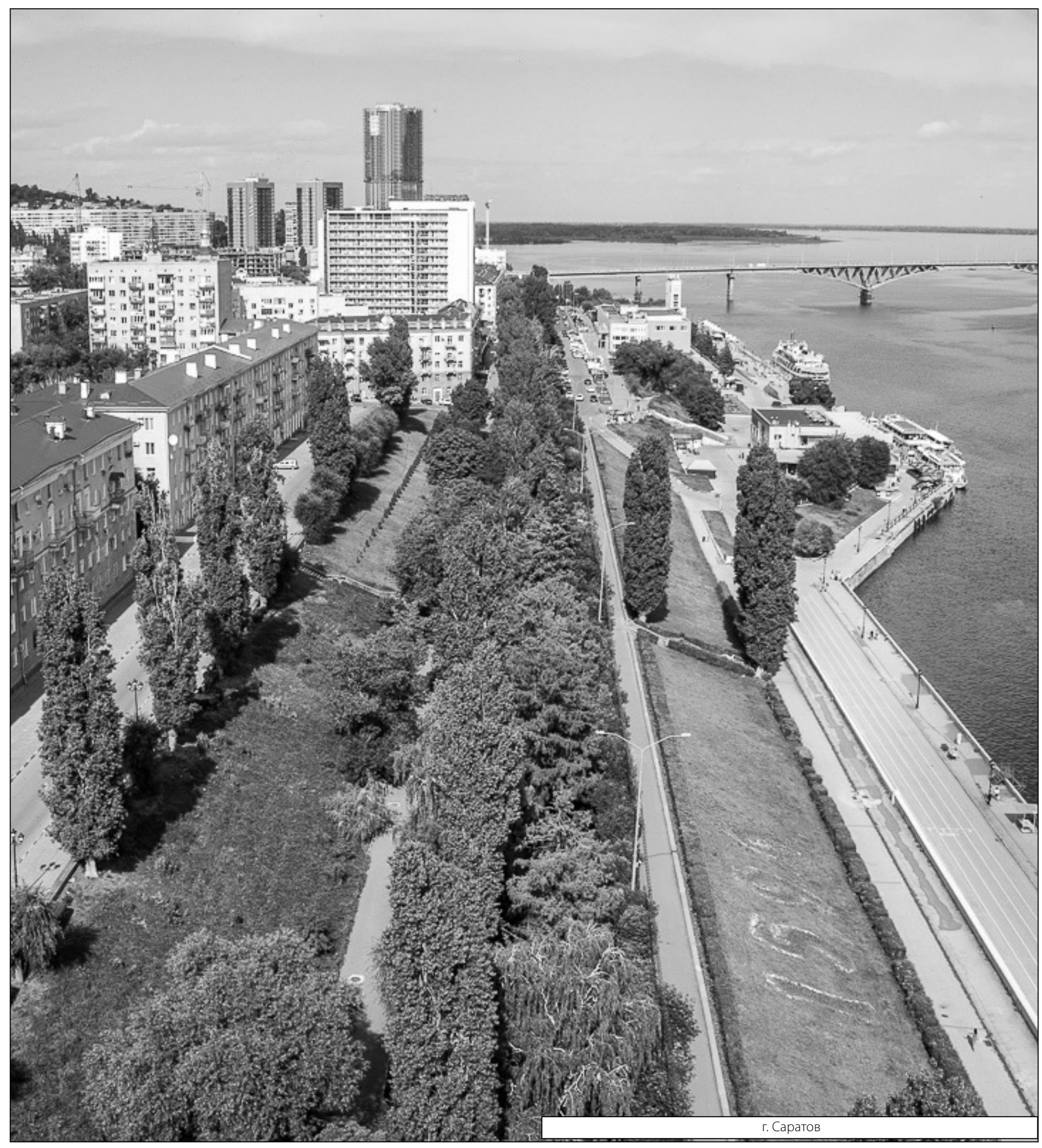

